\title{
Digital Image Restoration for Phase-Coded Imaging Systems
}

\author{
Chen-Yu Tseng *, Sheng-Jyh Wang*, Chir-Weei Chang**, Po-Chang Chen**, \\ Chuan-Chung Chang**, Yi-An Chen* \\ * Dept. of Electronics Engineering, National Chiao Tung Univ., \\ 1001 Ta-Hsueh Road Hsinchu,Taiwan, R.O.C. \\ ** Optoelectronics Research Laboratories, Industrial Technology Research Institute Research \\ 195, Sec. 4, Chung Hsing Rd., Chutung, Hsinchu, Taiwan 31040. R.O.C.
}

\begin{abstract}
This paper proposes a digital image restoration algorithm for phase-coded imaging systems. In order to extend the depthof-field (Dof), an imaging system equipped with a properly designed phase-coded lens can achieve an approximately constant point spread function (PSF) for a wide range of depths. In general, a phase-coded imaging system produces blurred intermediate images and requires subsequent restoration processing to generate clear images. For lowcomputational consumer applications, the kernel size of the restoration filter is a major concern. To fit for practical applications, a pyramid-based restoration algorithm is proposed in which we decompose the intermediate image into the form of Laplacian pyramid and perform restoration over each level individually. This approach provides the flexibility in filter design to maintain manufacturing specification. On the other hand, image noise may seriously degrade the performance of the restored images. To deal with this problem, we propose a Pyramid-Based Adaptive Restoration (PBAR) method, which restores the intermediate image with an adaptive noise suppression module to improve the performance of the phase-coded imaging system for Dof extension.
\end{abstract}

Keywords: Keywords: Phase-coded imaging, image restoration, deblurring

\section{INTRODUCTION}

In recent years, imaging systems equipped with properly designed phase-coded lens have been proposed to extend the depth-of-field (DoF). In [1] and [2], Dowski and Cathey proposed wave-front coding optics to make the point-spread function (PSF) and the optical transfer function approximately invariant to misfocus. Inspired by wave-front coding, Sung et al. designed new phase-coded optics for applications of mobile phone lens in [3] to reduce the focus-related aberration. In general, these phase-coded imaging systems produce intermediate images which are constantly blurred due to the corresponding focus-invariant PSF, and image restoration is required to generate clear output images.

The process of image restoration can be seen as a decoding process or a computational software lens to compensate the blurring PSF of the actual optical system. However in practice, it is difficult to estimate the actual optical PSF of the imaging system due to the manufacturing inaccuracy. In [4], Chen et al. developed a flow to design restoration filters with no need of the optical PSF information. In that approach, they took a shot of a well-designed test chart with the extended DoF (EDoF) camera to obtain the blurred image. Since the original test chart is known as the target image, a minimum mean-square-error (MMSE) restoration filter can be obtained based on the target image and the blurred image.

However, for applications concerning computational cost, such as mobile cameras, a large-size restoration filter is not practical. Moreover, noise boosting is a serious problem that always degrades the quality of the restored images. In this paper, we propose a Pyramid-Based Adaptive Restoration (PBAR) method, which decomposes the intermediate image into the form of Laplacian pyramid and restores each level individually. In this pyramid scheme, different levels refer to different frequency bands. For the coarse level, the computational complexity is low and we perform precise restoration to achieve better visual quality. On the other hand, since the inaccuracy of restoration in higher frequency bands may not cause noticeable artifacts for human visual perception, we adopt a less precise reconstruction filter to reduce the computational cost. Besides, since noise in different levels may cause different perceptual influence, we also propose a level-variant noise model to achieve adaptive noise suppression at different pyramid levels during the restoration process.

Optics, Photonics, and Digital Technologies for Multimedia Applications, edited by Peter Schelkens,

Touradj Ebrahimi, Gabriel Cristóbal, Frédéric Truchetet, Pasi Saarikko, Proc. of SPIE Vol. 7723,

$77231 \mathrm{~N} \cdot$ @ 2010 SPIE · CCC code: $0277-786 \mathrm{X} / 10 / \$ 18 \cdot$ doi: $10.1117 / 12.854288$

Proc. of SPIE Vol. $772377231 \mathrm{~N}-1$ 
With this PBAR method, we can efficiently restore the blurred intermediate images with effective noise suppression. This improvement greatly enhances the performance of the phase-coded imaging system for Dof extension.

\section{PYRAMID BASED ADAPTIVE RESTORATION}

In this section, we first briefly introduce the procedure to estimate the restoration filter based on the Minimum MeanSquare-Error (MMSE) restoration algorithm. We further extend this MMSE restoration process to a pyramid-based scheme to reduce the kernel size of the restoration filters. Finally, the proposed Pyramid-Based Adaptive Restoration (PBAR) is described which can effectively avoid the noise boosting problem during restoration and may improve the performance of the phase-coded imaging system.

\subsection{Restoration Filter Estimation}

In [4], Chen et al. developed a flow for designing restoration filters with no need of the optical PSF information, as illustrated in Figure 1. They first prepared a well-designed test pattern and captured it with the phase-coded imaging system to get a blurred intermediate image. Since the target image (known test pattern) and the blurred image are given, the Minimum Mean-Square-Error (MMSE) estimation is performed to construct the restoration filter, which is used to restore all blurred images of their imaging system.

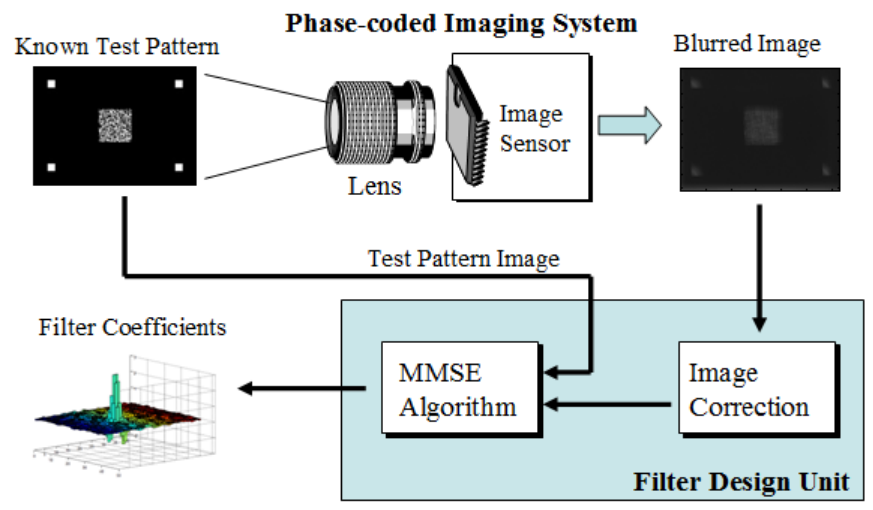

Figure 1. Design flow of MMSE filter.

To introduce the MMSE filter, we first model the optical channel as a linear shift-invariant system:

$$
B(i, j)=\sum_{k=1}^{P} \sum_{l=1}^{Q} I(i+k, j+l) H(k, l)+N(i, j),
$$

where $I$ and $B$ denote the original image (known test pattern) and blurred image, respectively, $H$ is the corresponding point-spread function in dimensions $P$ and $Q$, and $N$ is the additive noise. The general restoration process can be expressed as:

$$
\hat{I}(i, j)=\sum_{k=1}^{M} \sum_{l=1}^{N} B(i+k, j+l) W(k, l)
$$

$\hat{I}$ is the restored image with the restoration filter $W$ in dimensions $M$ and $N$. In order to make the output image close to the original target image, the objective function $J$ is defined based on the mean-square-error expectation: 


$$
\begin{aligned}
J & \equiv E\left\{(I(i, j)-\hat{I}(i, j))^{2}\right\} \\
& =E\left\{I^{2}(i, j)\right\}-2 E\{I(i, j) \hat{I}(i, j)\}+E\left\{\hat{I}^{2}(i, j)\right\} .
\end{aligned}
$$

To minimize the mean square error, partial derivative is perform over $J$ with respect to $W(k, l)$. To simplify the problem, we assume the noise component is independent of the original image and the partial derivative can be deduced as below:

$$
\frac{\partial J}{\partial W(k, l)}=-2 E\{I(i, j) B(i+k, j+l)\}+2 \sum_{p=1}^{M} \sum_{q=1}^{N} E\{B(i+p, j+q) B(i+k, j+l)\} W(p, q) .
$$

The minimum mean-square-error solution occurs when the partial derivative is equal to zero. That is, the MMSE filter can be calculated based on the following equations:

$$
\begin{aligned}
& R_{I B}(k, l)=\sum_{p=1}^{M} \sum_{q=1}^{N} R_{B B}(k-p, l-q) W(p, q) \\
& \text { where } R_{I B}(k, l)=E\{I(i, j) B(i+k, j+l)\} \\
& \text { and } R_{B B}(k-p, l-q)=E\{B(i+p, j+q) B(i+k, j+l)\} .
\end{aligned}
$$

Since the target image $I$ and the blurred image $B$ are given, the coefficient of the MMSE filer W can be calculated based on the deduction described in [4].

In (5), the parameters $M$ and $N$ correspond to the kernel size of the restoration filter. A more precise restoration requires a larger filter size. However, for practical applications, it is usually not acceptable to adopt a large-size filter. On the other hand, the actual noise component may not be truly independent of the original image. Sometimes, the image noise is more like a signal-dependent Poisson-Gaussian noise [5]. This signal-dependent noise conflicts with the assumption we applied in (4) and may cause apparent degradation in the quality of the restored image. To handle these two aforementioned problems, we propose a PBAR algorithm, which is to be introduced in following sections.

\subsection{Pyramid-Based Restoration (PBR) Filter}

In this section, we propose a design flow of the pyramid-based restoration. In this approach, we effectively reduce the kernel size of the restoration filter with only slight loss in perceptual quality. The flowchart is illustrated in Figure 2, where we first individually decompose the target image and the intermediate image into the form of Laplacian pyramid [6]. The iterative rule of the Gaussian-Laplacian pyramid method can be formulated as below, for $i$ increasing from 0 to N-1.

$$
\begin{gathered}
I_{i+1}^{\prime}=g \otimes I_{i}, \\
L_{i+1}=I_{i}-I_{i+1}^{\prime}, \\
I_{i+1}=\left(I_{i+1}^{\prime}\right)_{\downarrow 2} .
\end{gathered}
$$

In (6) (8), $I_{i}$ denotes the image of $i^{\text {th }}$ level of Gaussian pyramid, $I_{0}$ denotes the original image, $L_{i}$ denotes the residual image of $i^{\text {th }}$ level of the Laplacian pyramid, $g$ denotes a Gaussian filter, and $\downarrow 2$ denotes the down-sampling process.

After having decomposed the target image and the blurred image into the Laplacian pyramid representation, we extract level-pairs from both of these two pyramids level by level. Each level-pair contains one target image and one blurred image, denoted as $L_{i}^{T}$ and $L_{i}^{B}$, respectively, for $1 \leq i<N$ (residual levels). For $i=N$ (the top level), we denote the pair as $I_{N}^{T}$ and $I_{N}^{B}$. Applying the MMSE method for each level-pair individually, the level-restoration filter $w_{i}$ can be calculated for $1 \leq i \leq N$. 


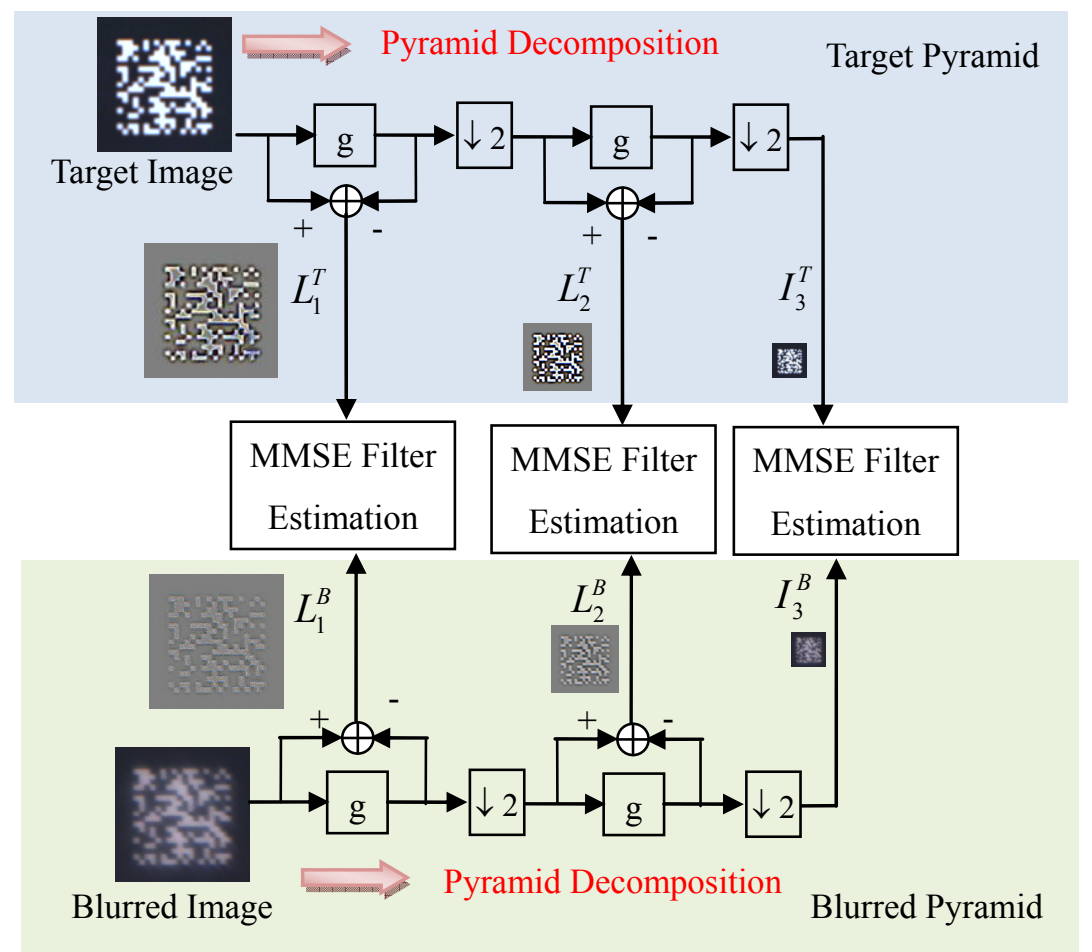

Figure 2. Design flow of a three-level pyramid-based restoration.

After the estimation of the MMSE restoration filters, the flowchart of this pyramid-based restoration is designed as illustrated in Figure 3. The intermediate image is decomposed into the form of Laplacian pyramid. After that, the levelbased restoration is performed for each level, successively from the top to the bottom of the pyramid. For the top level, the correspond restored layer $I_{N}^{R}$ can be derived by

$$
I_{N}^{R}=w_{N} \otimes I_{N}^{B} .
$$

For the subsequent levels (residual levels), the iterative rule can be formulated as

$$
\begin{gathered}
\hat{I}_{i}^{R}=g \otimes\left(I_{i+1}^{R}\right)_{\uparrow 2} \text { and } \\
I_{i}^{R}=\hat{I}_{i}^{R}+w_{i} \otimes L_{i}^{B}, \text { for } 0 \leq i<N,
\end{gathered}
$$

where $i$ is the level index decreasing from $N-1$ to 0 , and $I_{0}^{R}$ denotes the final output image.

In this pyramid scheme, different levels refer to different frequency bands. In this approach, the coefficients of the restoration process for each level can be adjusted to fit for manufacturing requirements. For the coarse level, the computational complexity is low due to the down-sampling processes in the pyramid computation. On the other hand, since the inaccuracy of restoration in higher frequency bands may not cause noticeable artifacts for human visual perception, we adopt a less precise reconstruction filter in the finer level to reduce the computational cost.

Figure 4 (b) shows the output image by directly using a $15 \times 15$ restoration filter. Figure 4 (c) shows the output image by applying the pyramid-based restoration with a $7 \times 7$ restoration filter for each level. Their perceptual performances are similar, but the proposed method effectively reduces the kernel size of the restoration filter. 


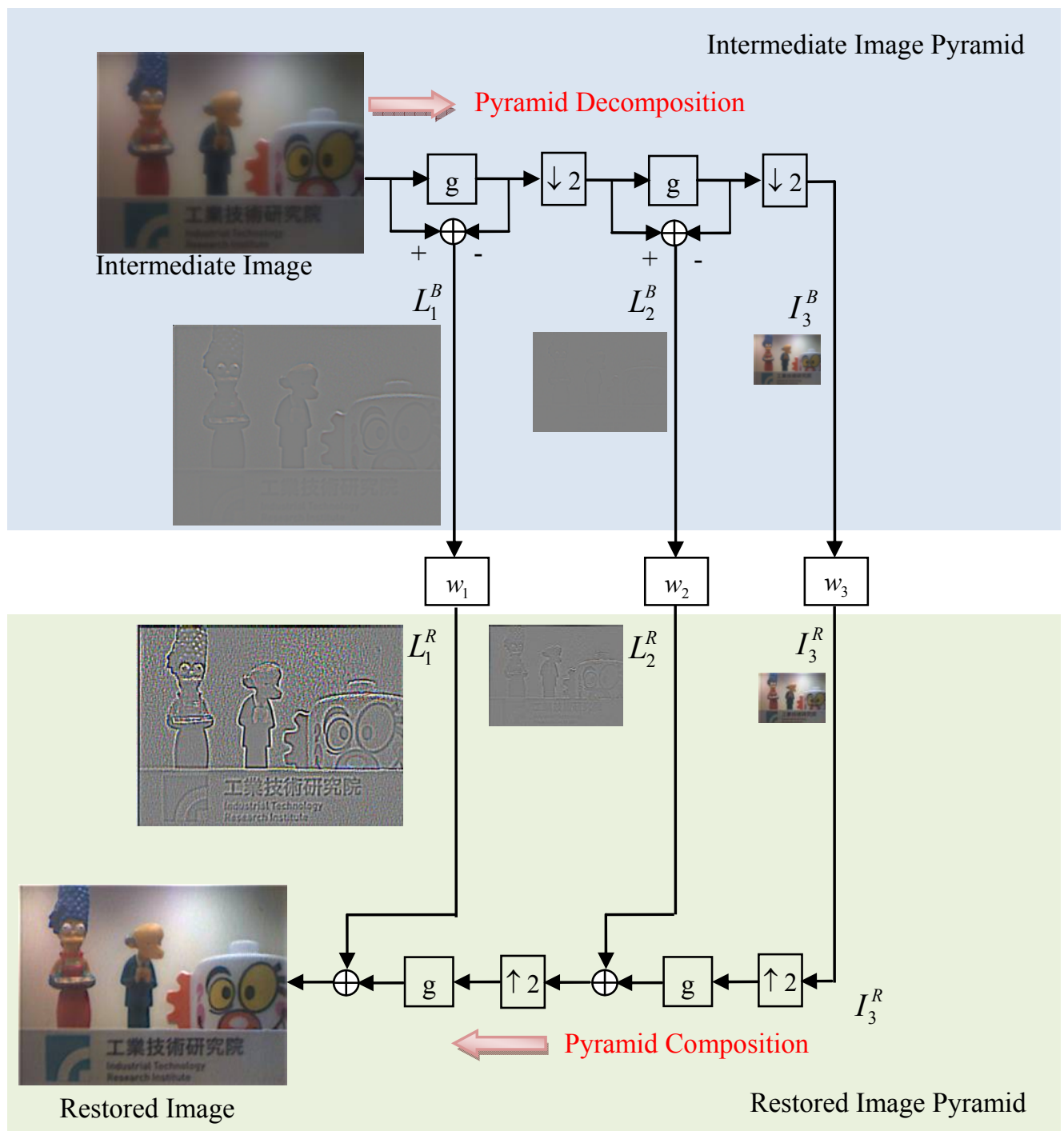

Figure 3. The flowchart of a three-level pyramid-based restoration. 


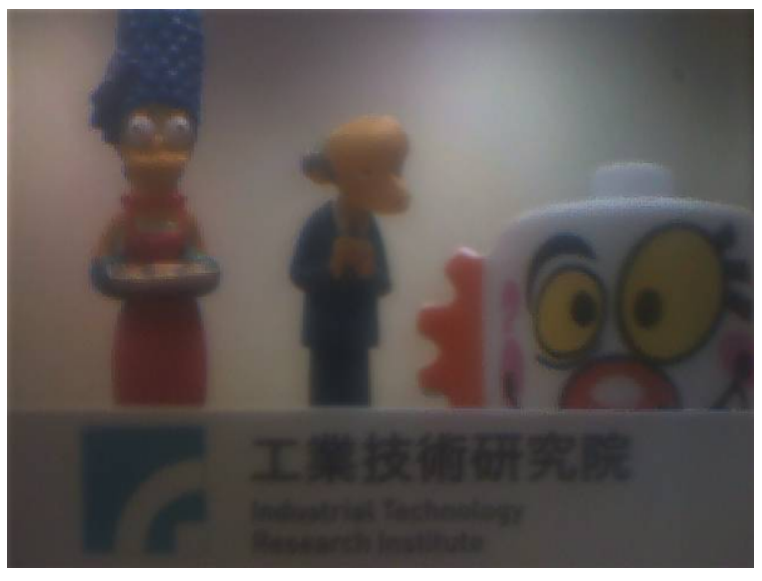

(a) Intermediate image

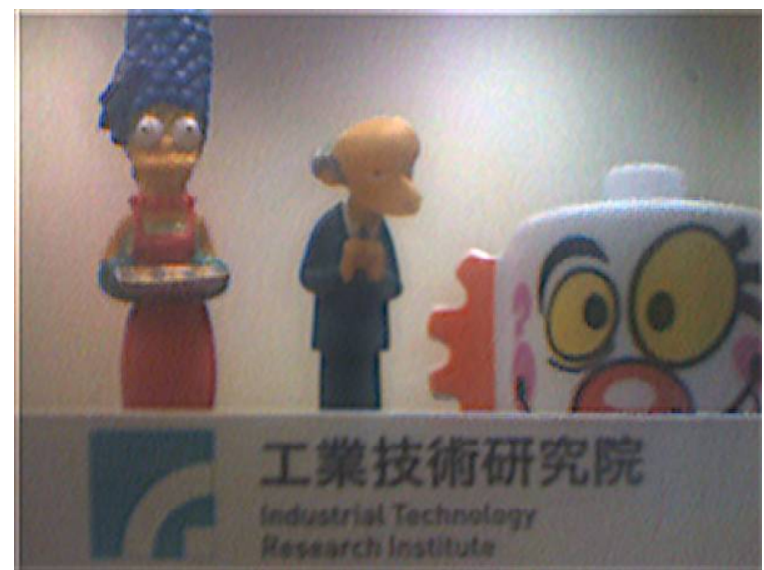

(b) Output image with a $15 \times 15$ restoration filer

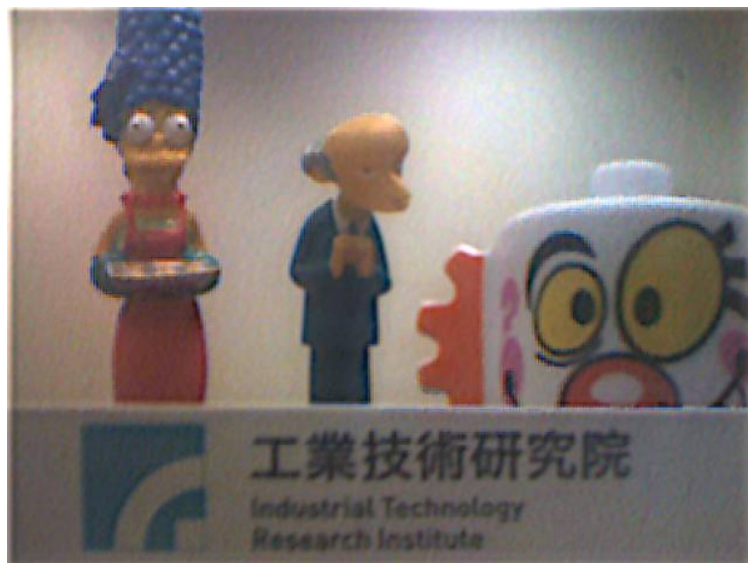

(c) Output image with a 3-level pyramid-based restoration,

$7 \times 7$ restoration filter for each level

Figure 4. (a) Original image, (b) output image with a $15 \times 15$ restoration filter, and (c) the result of pyramid-based restoration.

\subsection{Pyramid-Based Adaptive Restoration (PBAR) with Noise Suppression}

Image acquisition noise is always a serious problem for restoration. Image noise may seriously degrade the quality of the restored image. As mentioned above, the actual noise component may not be truly signal-independent [5]. This fact conflicts with the assumption adopted in the deduction of the MMSE restoration. To deal with this problem, a PyramidBased Adaptive Restoration (PBAR) is proposed. Based on the pyramid-based restoration introduced in the preceding section, the parameters in each level can be individually adjusted in the PBAR approach, depending on the corresponding noise level.

In [5] Foi et al. proposed a signal-dependent noise model for the raw data of image sensors. For each pixel, the signaldependent model represents the noise standard-deviation as a function of the expectation value of the pixel intensity. The observed signal is modeled as

$$
z(x)=y(x)+\sigma(y(x)) \xi(x)
$$


where $z$ is the observed pixel intensity at the position $x, y$ is the original intensity, $\sigma$ is a function of $y$, and $\xi$ is zeromean random noise with its standard deviation equal to 1. In [5] Foi et al. assumed that the noise term is composed of two parts: the signal-dependent Poisson component $\eta_{p}$ and the signal-independent Gaussian component $\eta_{g}$. That is,

$$
\sigma(y(x)) \xi(x)=\eta_{p}(y(x))+\eta_{g} .
$$

The variance of the Poisson component depends on the value of the original intensity $y$. This component follows the Poisson distribution and we have $\operatorname{var}\left\{\eta_{p}(y(x))\right\}=a y(x)$, with $a$ being a parameter corresponding to the sensor hardware. On the other hand, for the Gaussian component, the noise variance is a constant value $b$. The overall noise variance function can be represented in a linear form as

$$
\sigma^{2}(y(x))=a y(x)+b .
$$

In this noise model, the coefficients $a$ and $b$ depend only on sensor hardware. They can be estimated empirically [5]. Based on this noise model, a Pyramid-Based Adaptive Restoration (PBAR) is proposed as illustrated in Figure 5. Moreover, by adopting this noise model, we can also avoid the noise boosting problem during restoration.

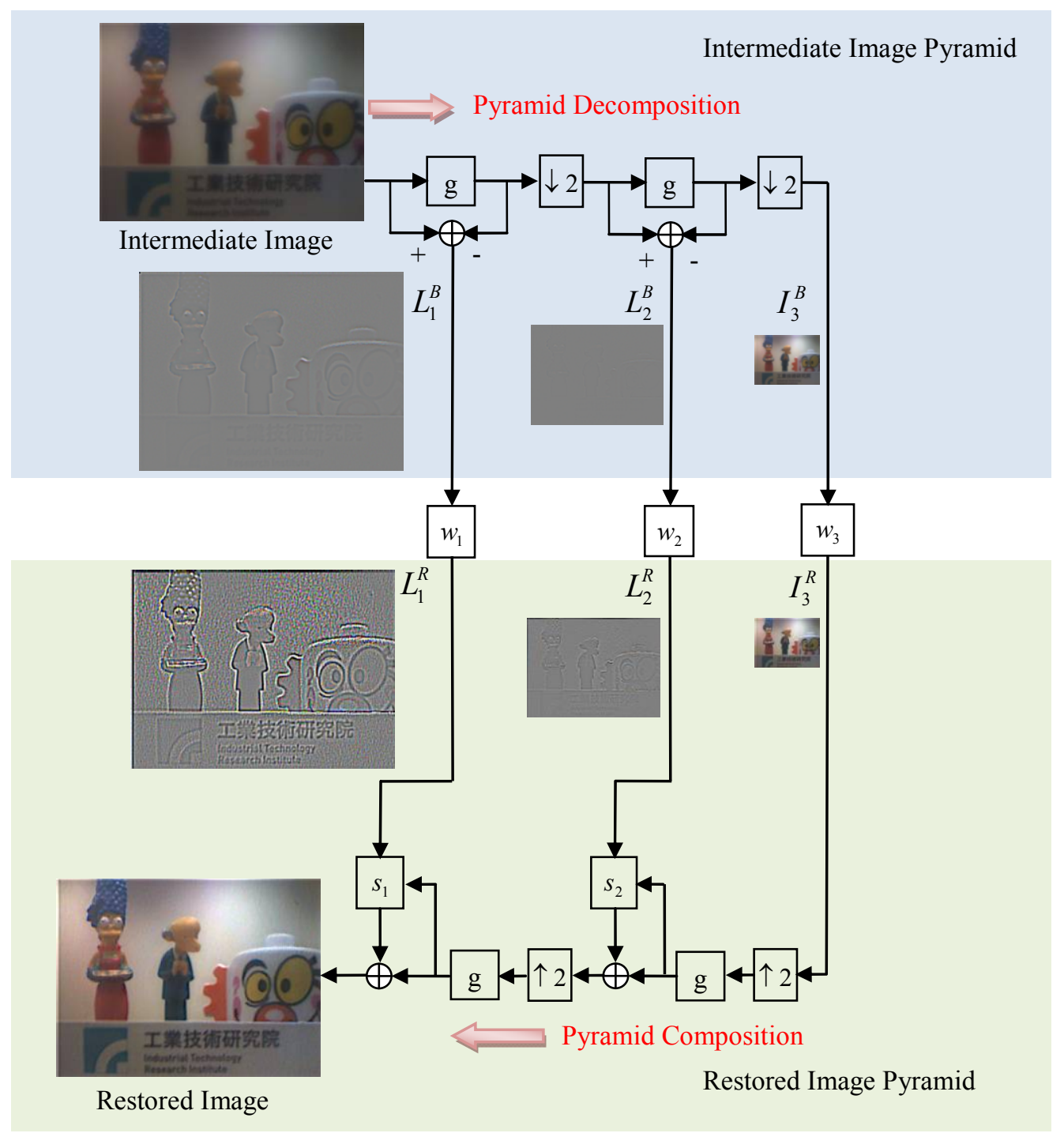

Figure 5. The flowchart of a three-level scheme of the pyramid-based adaptive restoration. 
In Figure 5, a three-level scheme of PBAR is illustrated where the noise suppression module is added after the levelrestoration for each residual level. Since the general noise model is signal-dependent as expressed in (14), our proposed adaptive noise suppression module is a function described as below:

$$
\begin{aligned}
\bar{L}_{i}^{R}(x) & =s_{i}\left(L_{i}^{R}(x), \hat{I}_{i}^{R}(x)\right) \\
& =\left\{\begin{array}{c}
L_{i}^{R}(x), \text { for }\left(L_{i}^{R}(x)\right)^{2}<t h_{i}\left(\hat{I}_{i}^{R}(x)\right) \\
0, \text { otherwise }
\end{array}\right.
\end{aligned}
$$

In (15), $\bar{L}_{i}^{R}(x)$ is the output residual value of the $i^{t h}$ level at the pixel position $x$. This value is produced from the noise suppression function $s_{i}\left(L_{i}^{R}(x), \hat{I}_{i}^{R}(x)\right)$ that removes the noise component involved in $L_{i}^{R}$, depending on the noisethreshold $t h_{i}\left(\hat{I}_{i}^{R}(x)\right)$, which is defined as

$$
\operatorname{th}_{i}\left(\hat{I}_{i}^{R}(x)\right) \equiv a_{i} \hat{I}_{i}^{R}(x)+b_{i} .
$$

In (16), $a_{i}$ and $b_{i}$ are noise parameters. This noise-threshold function is based on the noise model described in (14). The parameters depend on the corresponding sensor parameters that can be estimated based on Foi's method [5]. Furthermore, we directly use the intensity value of $\hat{I}_{i}^{R}(x)$ to approximate the original intensity value $y(x)$. This is because the noise component has mostly been removed through the sequent Gaussian filtering during the process of pyramid decomposition.

\section{EXPERMENTAL RESULTS}

The experiment platform is set as shown in Figure 6. Figure 6(a) is the imaging system containing an extended depth-offield (EDoF) camera and a personal computer for image restoration. The EDoF camera is equipped with a singlet coding lens without cover glass and IR cut, as shown in Figure 6 (b). The focal length is $4 \mathrm{~mm}$. Compared to the system with traditional lens, this phase-coding imaging system can effectively extend the depth of focus, as shown in Figure 7.

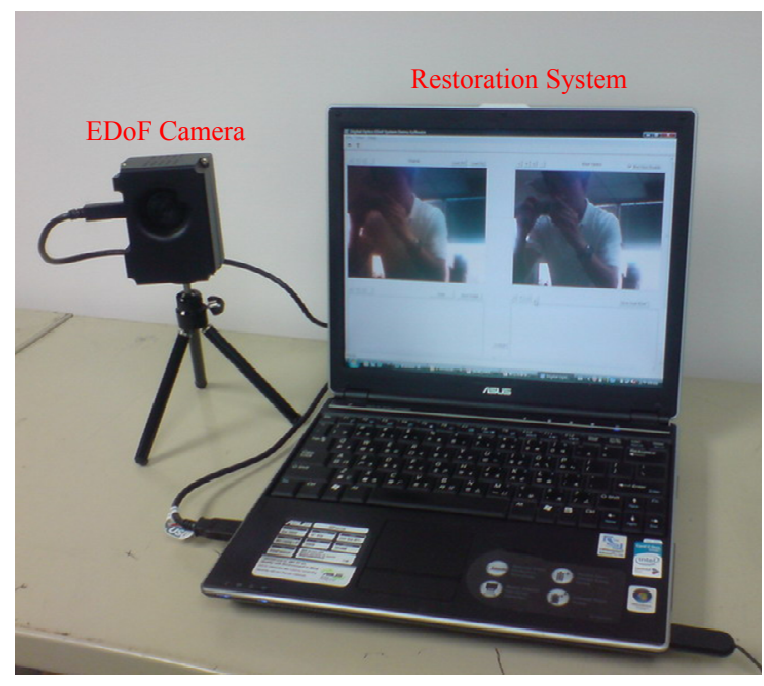

(a) Phase-coded Imaging System

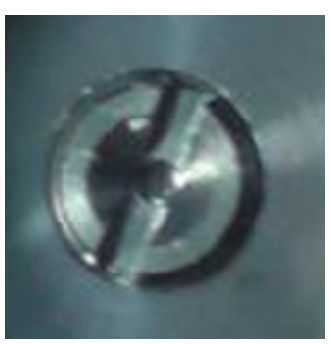

(b) Singlet Coding Lens

Figure 6. Experiment platform. 


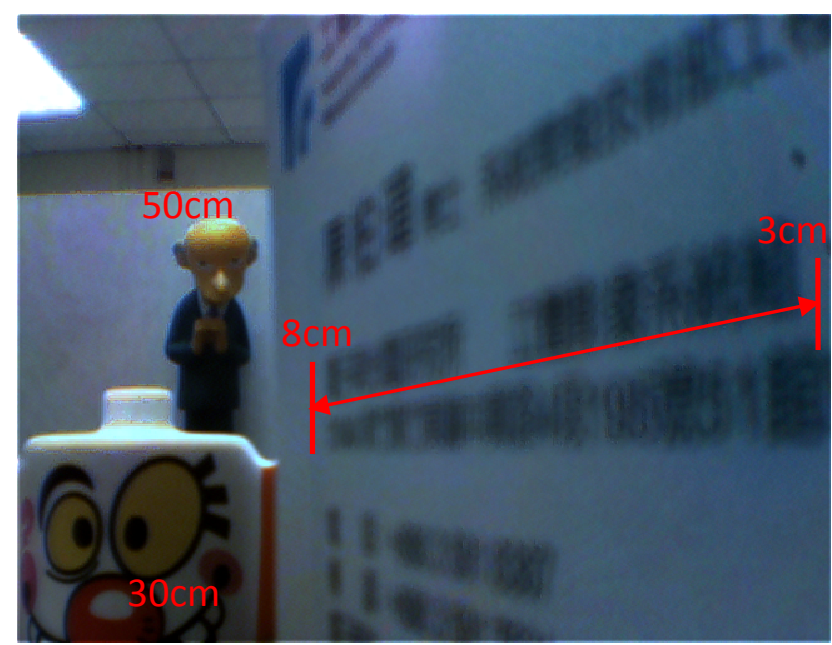

(a) Output Image of a Conventional Camera

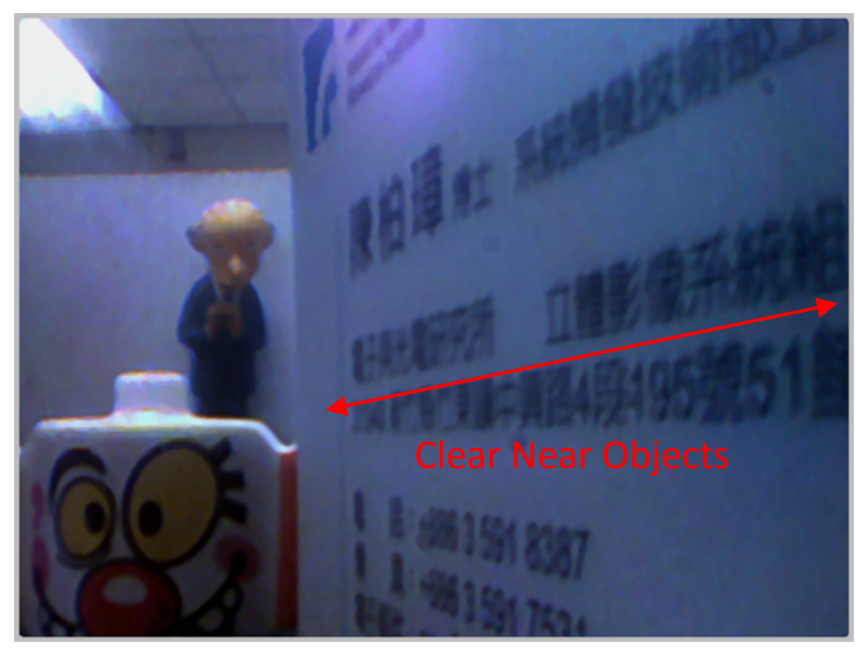

(b) Output Image of the EDoF Camera

Figure 7. Experiment platform.

The intermediate images produced from the EDof camera are further passed through the restoration process. The restoration results are shown in Figure 8 and Figure 9, which are the $2^{\text {nd }}$-level and the $1^{\text {st }}$-level residual images from the Laplacian pyramids of the intermediate image, the PBR output image, and the PBAR output image. The magnitude of these residual images is post-enhanced five times for easier observation. The enhanced results show that the noise boosting effect can be effectively reduced through the PBAR method. This PBAR method does successfully improve the performance of the phase-coded imaging system as shown in Figure 10.

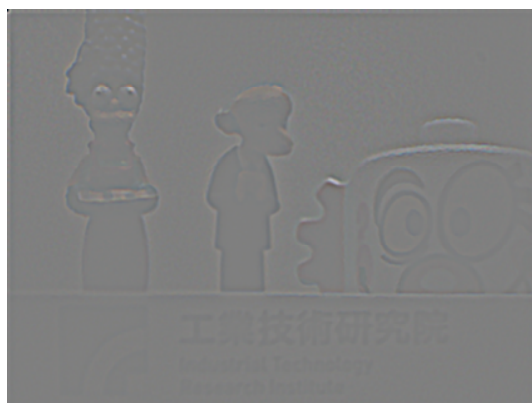

(a) Intermediate image, $L_{2}^{B}(x)$

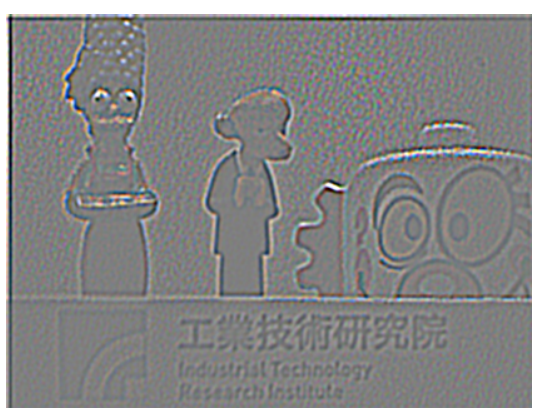

(b) Restored image, $L_{2}^{R}(x)$

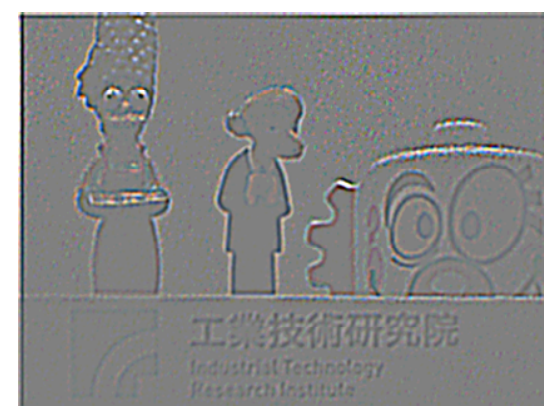

(c) Adaptive Restored image, $\bar{L}_{2}^{R}(x)$

Figure 8. (a)The $2^{\text {nd }}$-level intermediate residual image, and the output residual images of (b) PBR and (c) PBAR. 


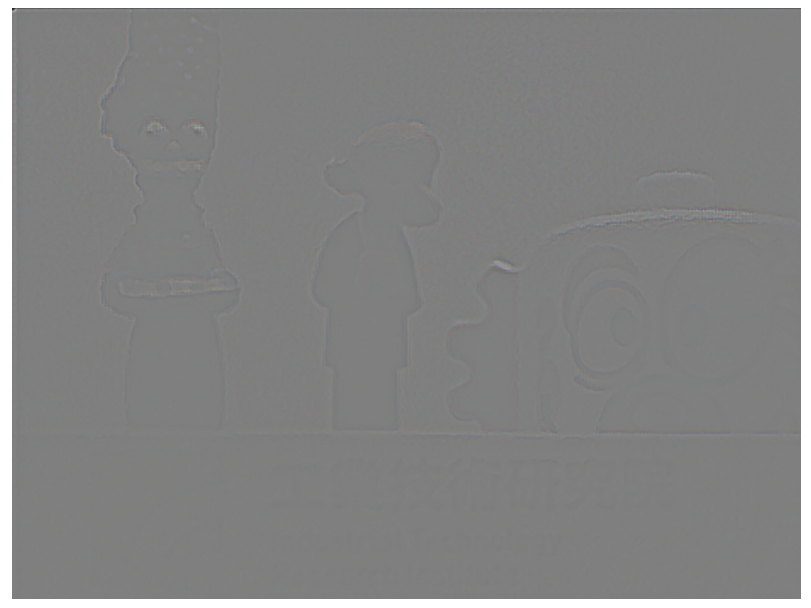

(a) Intermediate residual image, $L_{1}^{B}$

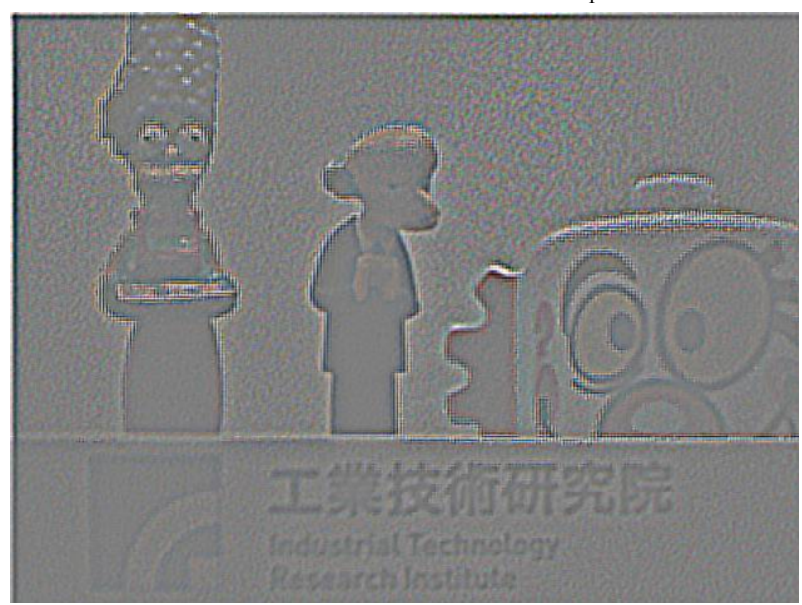

(b) Output residual image of PBR, $L_{1}^{R}$

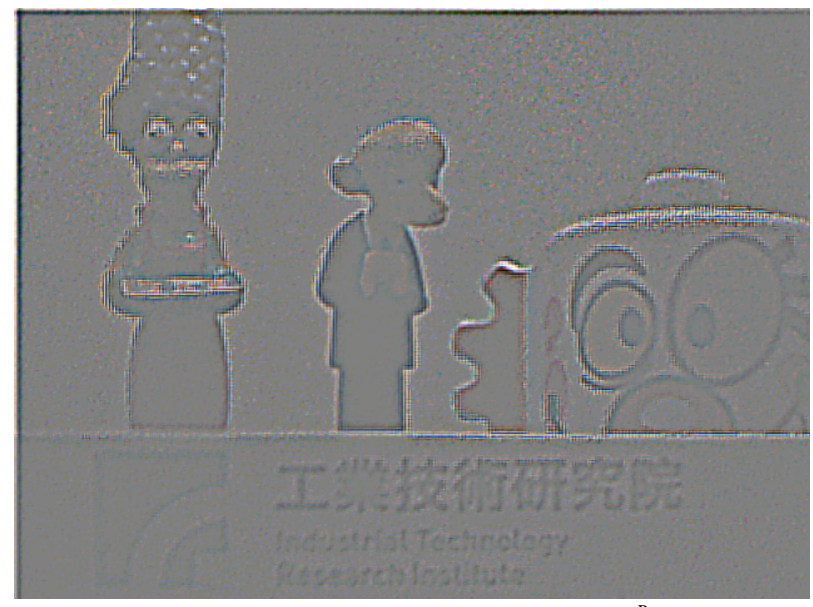

(c) Output residual image of PBAR, $\bar{L}_{1}^{R}$

Figure 9. (a)The $1^{\text {st }}$-level intermediate residual image, and the output residual images of (b) PBR and (c) PBAR.

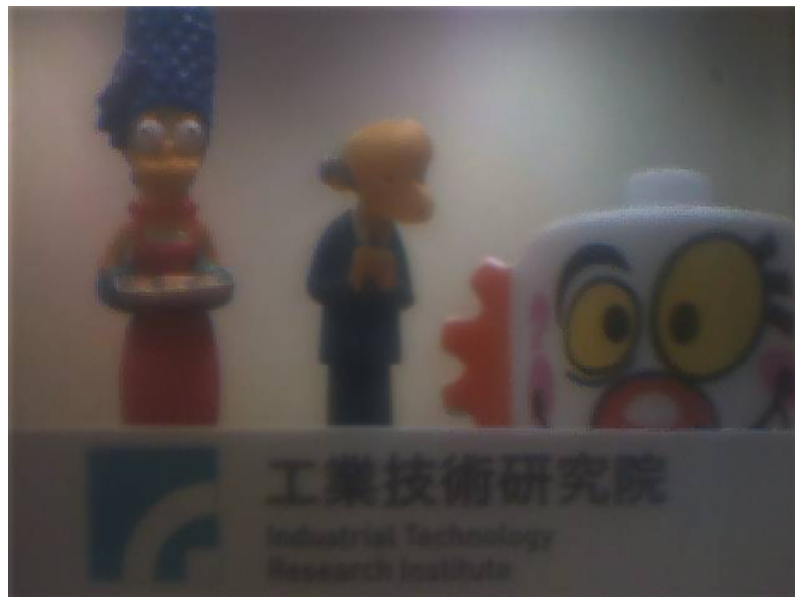

(a) Intermediate image

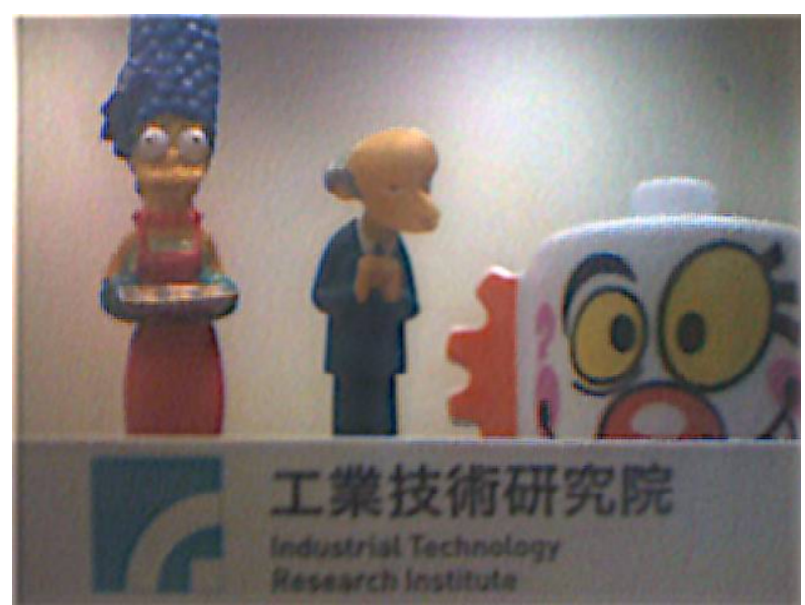

(b) Output residual image of PBR

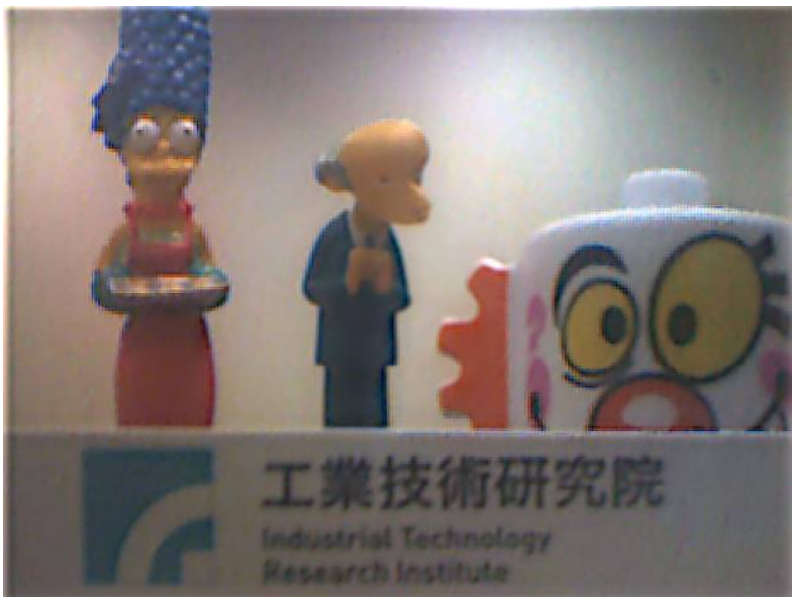

(c) Output residual image of PBAR

Figure 10. (a)The intermediate image, and the output images of (b) PBR and (c) PBAR. 


\section{CONCLUSIONS}

In this paper, a pyramid-based adaptive restoration algorithm for phase-coded imaging system is presented. In this approach, the blurred intermediate image is decomposed into the form of Laplacian pyramid and each level is restored individually. This approach provides the flexibility to adjust parameters at each level in order to fit for the design specification, such as kernel size of the restoration filter. Moreover this PBAR algorithm provides noise-suppressed results based on a signal-dependent noise model. This PBAR algorithm can successfully suppress the noise boosting problem and improve the output quality of the EDoF system.

\section{ACKNOWLEDGEMENT}

This research was supported by Ministry of Economic Affairs, R. O. C. under Grant Number 98-EC-17-A-02-S2-0047.

\section{REFERENCES}

[1] E. R. Dowski, and W. T. Cathey, "Extended depth of field through wave-front coding," Applied Optics 34(11), 1859-1866 (1995).

[2] W. T. Cathey and E. R. Dowski, "New paradigm for imaging systems," Applied Optics 41(29), 6080-6092 (2002).

[3] H. Y. Sung, S. S. Yang and H. Chang, "Design of mobile phone lens with extended depth of field based on pointspread function focus invariance,"Proc. SPIE 7061, 706107.1-706107.11 (2008).

[4] P. C. Chen, C. H. Liu, C. W. Chang, C. C. Chang, L. Angot, "Digital Decoding Design for Phase Coded Imaging System," Proc. of SPIE 7443, 74431A (2009).

[5] Foi, A., M. Trimeche, V. Katkovnik, and K. Egiazarian, "Practical Poissonian-Gaussian noise modeling and fitting for single-image raw-data", IEEE Trans. Image Process., 17(10), 1737-1754(2008).

[6] P.J. Burt et A.E. Adelson, "The Laplacian pyramid as a compact image code", IEEE Trans. on Communications 31, 532-540 (1983). 